\title{
Response to: Letter to the Editor: Long-Term Results of Laparoscopic Sleeve Gastrectomy for Morbid Obesity: 5 to 8-Year Results
}

\author{
Ralph P. M. Gadiot ${ }^{1} \cdot$ L. Ulas Biter ${ }^{1}$
}

Published online: 30 March 2017

(C) Springer Science+Business Media New York 2017

In response to the letter to the Editor:

We greatly appreciate the interest and comments on our article regarding long-term outcome after sleeve gastrectomy [1].

In response to your first question concerning weight loss failure and weight regain percentages, we consulted our database. Weight loss failure versus weight regain was $4.8 \%$ versus $10.9 \%$ at 5 years, $8.4 \%$ versus $15.4 \%$ at 6 years, $7.7 \%$ versus $9.2 \%$ at 7 years, and $13.3 \%$ versus $16.7 \%$ at 8 years.

For your second question, we analyzed the data of our patients requiring revision to laparoscopic roux-and-Y gastric bypass for inadequate weight loss $(n=38)$. A total of 22 patients needed revision for weight loss failure versus 16 patients for weight regain. Successful revision, defined as achieving at least 50\% excess weight loss from index weight, following revision to LRYGB was achieved in 7 patients $(32 \%)$ in the weight loss failure group versus 11 patients (73\%) in the weight regain group. This difference between both groups is a very interesting finding and should be investigated. This subdivision of failed sleeve gastrectomy in weight loss failure versus weight regain has not been described in literature to our knowledge.

The results of this study had no specific implications on our patient selection for either LSG or LRYGB. The same patient

Ralph P. M. Gadiot

r.gadiot@franciscus.nl; r.gadiot@sfg.nl

L. Ulas Biter

u.biter@franciscus.nl

1 Franciscus Gasthuis \& Vlietland, Kleiweg 500, 3045

PM Rotterdam, The Netherlands characteristics and patient preference are still used in our decision-making. One of the reasons that we still perform both procedures in roughly the same ratio is the randomized controlled "sleeve versus bypass trial" which is performed in our institution [2].

\section{Compliance with Ethical Standards}

Conflict of Interest The authors declare that they have no conflict of interest.

Ethical Approval This article does not contain any studies with human participants or animals performed by any of the authors.

Informed Consent Does not apply

\section{References}

1. Gadiot RP, Biter LU, van Mil S, et al. Long-term results of laparoscopic sleeve gastrectomy for morbid obesity: 5 to 8 -year results. Obes Surg. 2017;27(1):59-63.

2. Biter LU, Gadiot RP, Grotenhuis BA, et al. The Sleeve Bypass Trial: a multicentre randomized controlled trial comparing the long term outcome of laparoscopic sleeve gastrectomy and gastric bypass for morbid obesity in terms of excess BMI loss percentage and quality of life. BMC Obes. 2015;2:30. 